\section{Impeccable treatment for your patient}

Right now, things are still highly unprecedented and stressful in many dental practices. If you are dealing with an urgent or complex case, consider referring your patient to the Centre for Oral-Maxillofacial and Dental Implant Reconstruction.

Led by Professor Cemal Ucer Specialist Oral Surgeon - the cuttingedge treatment centre offers a wide variety of oral surgery treatments such as surgical removal of teeth, cystic lesions and pathology as well as advanced dental and oral implantology procedures, major bone grafting, including zygomatic dental implants for the rehabilitation of severely atrophic patients. It features cutting-edge facilities such as a day care operating theatre and recovery suite, in house digital laboratory and digital diagnostic imaging centre, and has a clinical team that boasts considerable experience, ensuring they are well equipped to handle demanding, high-risk and urgent cases.

One thing you can be certain of is the team's dedication to giving your patient impeccable treatment. Refer today.

Contact Professor Ucer at ice@ucer.uk or Mel Hay at mel@mdic.co. Call 01612 371842 .

\section{A colourless universal composite}

Tokuyama are the only composite manufacturer to use patented spherical filler particles within their composite materials. Each variant utilising spherical particles of different diameters to maximise their optical and physical properties for the desired indication.

In addition to optimised optical properties resulting in enhanced aesthetic restorations, Tokuyama's spherical filler particles offer other significant advantages compared with the irregular shaped filler particles used by all other manufacturers. They are quicker and easier to pack into nooks and crannies, reducing the risk of voids; easier to sculpt and carve, producing enhanced morphology of the final restoration; and a much smoother surface finish which has a natural high sheen that requires minimal if any polishing.

Tokuyama's development of spherical filler particles has culminated in Omnichroma, the world's only colourless universal composite. It is the only universal composite that can match every tooth colour no matter what shade of white.

Now Tokuyama have launched low viscosity Omnichroma Flow, specifically to meet the needs of clinicians who prefer

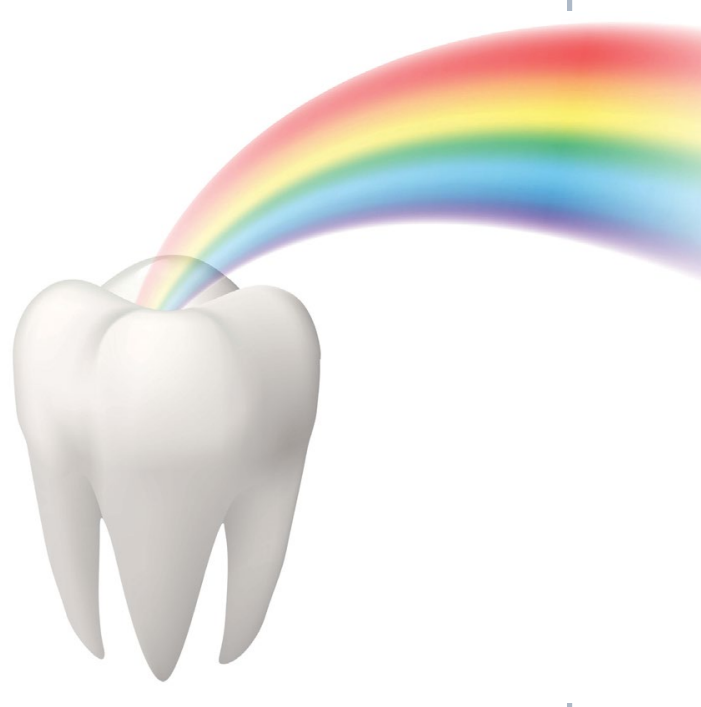

flowable materials in certain clinical situations - anterior and posterior restorations, cavity bases or lining and composite/ceramic repairs. It offers the benefits of perfect colour matching whatever the shade of tooth, simplified inventory management, excellent cavity adaptation, non-sticky, excellent polishability, remarkable glossiness and freedom from BisGMA and bisphenol A-related monomers.

For more information about the complete Tokuyama range, including Omnichroma Flow, contact your local Trycare Representative, call 01274885544 or visit www.trycare.co.uk.

\title{
New implants with excellent primary stability
}

Neoss ProActive Edge is a one-of-a-kind implant - an empowering addition to the Neoss implant family, giving you outstanding primary stability even in soft or compromised bone.

Data from a recent EAO study show that the Neoss ProActive Edge implants achieve equal or better implant stability than the top premium brand implants. In addition, Neoss ProActive Edge was the only implant that maintained outstanding implant stability in all situations, even with over-prepared osteotomies, making it highly predictable to use. $^{1}$

A revolutionary 1 to 2 -step drilling protocol prepares the site with minimal bone removal, giving you immediate feedback during placement. The drills are directly matched to the implant and thread designs, making placement faster, simpler and reducing chair time for you and your patients.

The predictability of the Neoss ProActive Edge implant has been further confirmed in a clinical use study. High primary stability was achieved for all implants, allowing for immediate or early load in support of single crowns, partial bridges, as well as for full arch restorations. All implants remained in function during the entire one-year observation period with minimal ( $-0.5 \mathrm{~mm})$ bone remodelling. ${ }^{2}$

Like all Neoss implants, Neoss ProActive Edge features the clinically proven super hydrophilic ProActive surface and the highly trusted NeoLoc connection.

One prosthetic platform across three implant ranges with one screwdriver connection! That is Intelligent Simplicity!

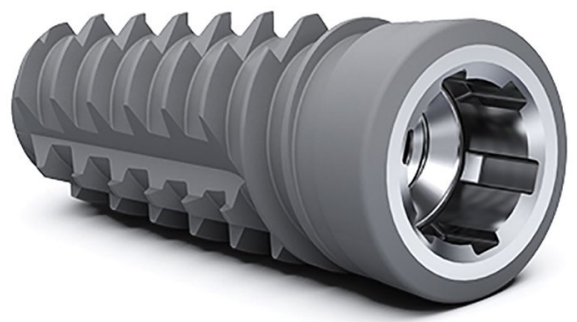

To find out more about the Neoss ProActive Edge implant visit www.neoss. com/edge or email info@neoss.com.

\section{References}

1. Turra M, Petersson A, Sennerby L. Primary stability of three different aggressively threaded and tapered implants in low-density polyurethane. COIR 2020; 31: 60.

2. Turra M, Andersson P, Verrocchi D, Sennerby L. A retrospective case series report on a novel aggressively threaded and tapered implant. COIR 2020; 31: 257. 